\title{
ARTICLE
}

\section{Shielding Experiments at High Energy Accelerators of Fermilab (III): Neutron Spectrum Measurements in Intense Pulsed Neutron Fields of The 120-GeV Proton Facility Using A Current Bonner Sphere Technique}

\author{
Masayuki HAGIWARA ${ }^{1 *}$, Toshiya SANAMI ${ }^{1}$, Yosuke IWAMOTO ${ }^{2}$, Hiroyuki ARAKAWA ${ }^{3}$, Nobuhiro SHIGYO $^{3}$, \\ Nikolai MOKHOV ${ }^{4}$, Anthony LEVELING ${ }^{4}$, David BOEHNLEIN ${ }^{4}$, Vaziri KAMRAN ${ }^{4}$, Takashi NAKAMURA ${ }^{5,6}$, \\ Koji OISHI $^{6}$, Hideo HIRAYAMA ${ }^{1}$, Hiroshi NAKASHIMA ${ }^{2}$, Yukio SAKAMOTO ${ }^{2}$ and members of JASMIN corroboration \\ ${ }^{1}$ High Energy Accelerator Research Organization, 1-1, Oho, Tsukuba, Ibaraki 305-0801, Japan \\ ${ }^{2}$ Japan Atomic Energy Agency, Tokai, Naka, Ibaraki 319-1195, Japan \\ ${ }^{3}$ Kyushu University, 744, Motooka, Nishi-ku, Fukuoka 819-0395, Japan \\ ${ }^{4}$ Fermilab, Batavia, IL 60510-5011, USA \\ ${ }^{5}$ Tohoku University, Aoba, Aramaki, Aoba-ku, Sendai, Miyagi 980-8578, Japan \\ ${ }^{6}$ Shimizu Corporation, 4-17, Etchujima, 3-chome, Koto-ku, Tokyo 135-8530, Japan
}

\begin{abstract}
Neutron energy spectra behind iron and concrete shields have been measured at the anti-proton production target station in Fermi National Accelerator Laboratory to provide shielding benchmark data of neutrons produced from $120-\mathrm{GeV}$ proton induced reaction. To measure neutron spectra in the radiation bursts accompanied by the injection of intense pulsed proton beam into the target, we have developed a current readout technique of a multi-moderator spectrometer. We employed a pair of $\mathrm{BF}_{3}$ proportional counters filled with different ${ }^{10} \mathrm{~B}$ isotope enrichment gases, ${ }^{\text {nat }} \mathrm{BF}_{3}\left(18 \%\right.$ enrichment) and ${ }^{10} \mathrm{BF}_{3}(96 \%$ enrichment) and used them as current readout ionization chambers to avoid signal pile-up problem. The spectrometer is aimed to discriminate neutrons from other particles such as $\gamma$-rays and muons incoming in short bursts by subtracting results of the ${ }^{\text {nat }} \mathrm{BF}_{3}$ counter from that of the ${ }^{10} \mathrm{BF}_{3}$ counter. The neutron spectrum obtained with the present technique is generally consistent with results of neutron-induced activation measurements.
\end{abstract}

KEYWORDS: neutron energy spectra, multi-moderator spectrometer, radiation bursts, shielding benchmark data

\section{Introduction}

Estimation of neutron production and transmission through shielding are very important at high energy accelerator facilities because neutrons have high penetrability through shielding and large contribution to the external dose. A number of shielding design of high energy accelerator facilities are performed using particle interaction and transport codes such as MCNPX, FLUKA, MARS, PHITS and so on. ${ }^{1-4)}$ These codes should be assessed based on benchmark measurements to ensure the calculation results. However, benchmark data on neutron energy spectra behind shields induced by high energy protons above $1 \mathrm{GeV}$ are few because of lack of suitable facilities with a simple shielding structure and difficulties of neutron spectrum measurements especially for radiation bursts accompanied by beam operation with short pulse. $^{5-9)}$

We have performed series of shielding experiments at the anti-proton production target facility (pbar target station) in FNAL to measure the induced activities and neutron flux around high energy accelerators in the Japanese and American Study of Muon Interaction and Neutron detection (JASMIN) collaboration. ${ }^{10-12)}$ The pbar target station has developed to provide anti-protons to the proton - anti-proton collider (TEVATRON) using $120 \mathrm{GeV}$ protons hitting the

*Corresponding Author, E-mail:hagi@post.kek.jp

(C) Atomic Energy Society of Japan target. This facility has a simple structure above the target and beam dump and source term can be clearly defined. On the other hand, intense pulsed proton beam impacted on the target with power of about $65 \mathrm{~kW}$ and pulse width of several micro seconds produce radiation bursts even above thick shields, which make it difficult to apply active counters to measurements of the neutron spectrum.

In this study, we have developed a current readout Bonner sphere technique to measure neutron spectra in radiation bursts at the pbar target station by using a multi-moderator spectrometer (Bonner spheres) using a pair of $\mathrm{BF}_{3}$ proportional counters filled with different ${ }^{10} \mathrm{~B}$ isotope enrichment gases, ${ }^{\text {nat }} \mathrm{BF}_{3}\left(18 \%\right.$ enrichment) and ${ }^{10} \mathrm{BF}_{3}(96 \%$ enrichment). The combination of different ${ }^{10} \mathrm{~B}$-enriched $\mathrm{BF}_{3}$ counters allow to discriminate the signals of neutrons from other particles such as $\gamma$-rays and muons by subtracting results of the ${ }^{\text {nat }} \mathrm{BF}_{3}$ counter from those of the ${ }^{10} \mathrm{BF}_{3}$ counter. The neutron energy spectra behind shields are deduced by a conventional unfolding technique. The measured neutron energy spectra were compared with the results of neutron-induced activation measurements by means of reaction rates of nuclides. ${ }^{11}$

\section{Method}

\section{Detector}

Figure 1 shows a schematic view of the spectrometer. The spectrometer consists of two 2-inch-diameter $\mathrm{BF}_{3}$ 
spherical proportional counters (LND 2708) and four spherical polyethylene moderators with different diameters. One of these $\mathrm{BF}_{3}$ counters is filled with $96 \%{ }^{10} \mathrm{~B}$-enriched $\mathrm{BF}_{3}$ gas $\left({ }^{10} \mathrm{BF}_{3}\right)$ and the other is $18 \%{ }^{10} \mathrm{~B}$-enriched $\mathrm{BF}_{3}$ gas $\left({ }^{\text {nat }} \mathrm{BF}_{3}\right)$. The gas pressure of these $\mathrm{BF}_{3}$ counters is 760 Torr. The difference of outputs from ${ }^{10} \mathrm{BF}_{3}$ counter and ${ }^{\text {nat }} \mathrm{BF}_{3}$ counter is then considered to be only due to neutrons, since ${ }^{10} \mathrm{~B}$ has a much higher capture cross-section to low-energy neutrons than that of the other stable isotope ${ }^{11} \mathrm{~B}$.

The spherical moderators are made of polyethylene (density of $0.928 \mathrm{~g} / \mathrm{cm}^{3}$ ) and their diameters are $81,110,150$, $230 \mathrm{~mm}$. These moderators were originally designed by Uwamino et al. to use with a $5 \mathrm{~atm}{ }^{3} \mathrm{He}$ proportional counter. 13) The ${ }^{3} \mathrm{He}$ counter was replaced with ${ }^{10} \mathrm{BF}_{3}$ counter for this study to increase output charge produced in the neutron reaction owing to the higher Q-value of the ${ }^{10} \mathrm{~B}(\mathrm{n}, \alpha){ }^{7} \mathrm{Li}$ reaction $(2.79 \mathrm{MeV})$ than that of the ${ }^{3} \mathrm{He}(\mathrm{n}, \mathrm{p})^{3} \mathrm{H}$ reaction $(0.764 \mathrm{MeV})$, which is important to use it as a current readout ionization chamber described in later.

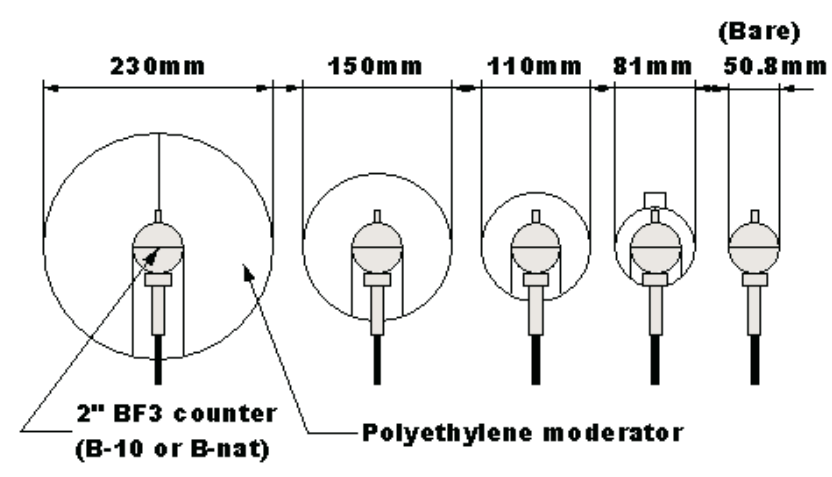

Fig. 1 The multi-moderator spectrometer used in this experiment. The spectrometer system has ten setups consist of two $\mathrm{BF}_{3}$ counters $\left({ }^{10} \mathrm{BF}_{3}\right.$ and $\left.{ }^{\text {nat }} \mathrm{BF}_{3}\right)$ and five moderator patterns $(0,81,110,150,230 \mathrm{~mm})$

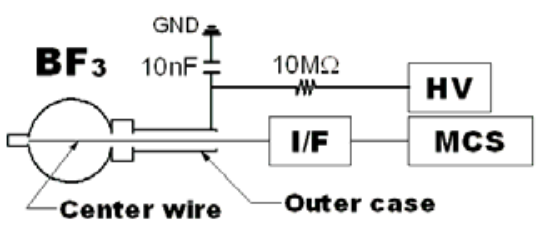

Fig. 2 Electronics of the current readout mode for a $\mathrm{BF}_{3}$ counter. High voltage is applied to the outer case of the $\mathrm{BF}_{3}$ counter to operate it as a current readout ionization chamber

\section{Signal readout}

Figure 2 shows a schematic diagram of electronics to operate the $\mathrm{BF}_{3}$ counter as a current readout ionization chamber. In the current readout mode, high voltage was applied to the outer case of the $\mathrm{BF}_{3}$ counter to read the current induced by ${ }^{10} \mathrm{~B}(\mathrm{n}, \alpha)^{7} \mathrm{Li}$ reaction on the central wire. The applied voltage was determined to be $300 \mathrm{~V}$, based on a measured saturation curve of the induced charge and leakage current. The current was fed to a current to frequency converter (I/F; Laboratory Equipment Co.), which converts input charge of $1 \mathrm{pC}$ to 1 pulse, and recorded by a multi-channel scaler (MCS) to measure the signals synchronized with the beam injection and monitor the fluctuation.

\section{Response functions}

Figure 3 shows the response functions for ${ }^{10} \mathrm{BF}_{3}$ counters with polyethylene moderators of $0,81,110,150,230 \mathrm{~mm}$ diameters calculated using the MCNPX code (version 25f) ${ }^{1)}$ with the evaluated cross-section data libraries. The library used for neutron transport and reaction rate were LA150 (hydrogen, carbon and stainless steel) and ENDF/B-VI (boron and fluorine) ${ }^{14)}$. For the energy region that is not covered by these libraries, MCNPX default physics models were used. The calculation was performed with mono-energetic parallel neutron beam of different energies from $10^{-9} \mathrm{eV}$ to $400 \mathrm{MeV}$ for each moderator diameter.

The response functions were measured with a conventional pulse readout system consists of a pre-amplifier and an amplifier and a multi-channel analyzer using $8.0 \mathrm{keV}$ monoenergetic neutrons at the FRS facility in Japan Atomic Energy Agency. ${ }^{15)}$ The agreement between experimental and calculated values was rather good as shown in Fig. 3. The shape of response functions in the current readout mode should not be so different with that in the pulse readout mode, because the both output signals are based on the measurement of the charge ionized by the outgoing particles from the ${ }^{10} \mathrm{~B}(\mathrm{n}, \alpha)^{7} \mathrm{Li}$ reaction. Iijima et al. report that the shape of the response function in a current readout mode agrees well with the calculation results of MCNPX as well as the response function in a pulse readout mode using a ${ }^{3} \mathrm{He}$ counter covered with polyethylene moderator. $^{16)}$

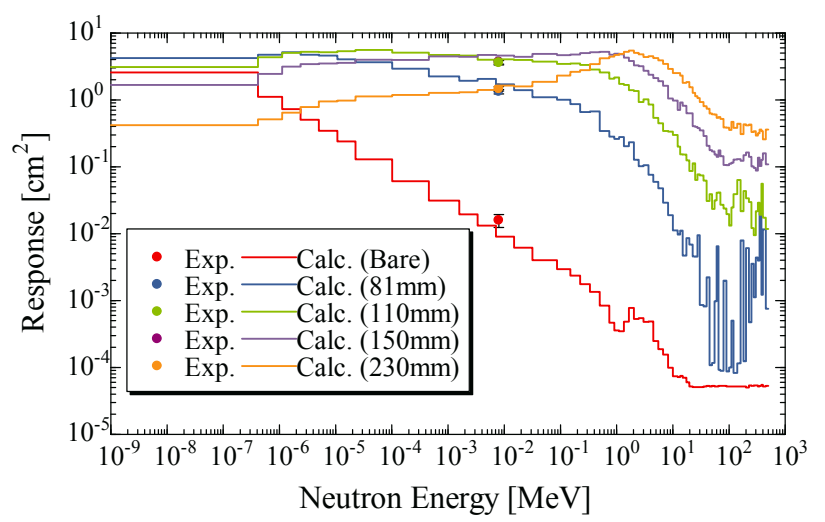

Fig. 3 Response functions of the ${ }^{10} \mathrm{BF}_{3}$ counter with fivemoderators for neutron energies from $10^{-3} \mathrm{eV}$ to $400 \mathrm{MeV}$

\section{Calibration}

To obtain the conversion factor from the current output (pC) to corresponding number of the ${ }^{10} \mathrm{~B}(\mathrm{n}, \alpha){ }^{7} \mathrm{Li}$ reaction in the $\mathrm{BF}_{3}$ counters, we irradiate a pair of ${ }^{10} \mathrm{BF}_{3}$ counters covered with the 230-mm-thick moderator using a neutron reference source of $3-\mathrm{Ci}^{241} \mathrm{Am}$-Be and measure the output counts per incident neutron in the pulse readout mode and the current readout mode. The ${ }^{241} \mathrm{Am}-\mathrm{Be}$ neutron source was placed $74 \mathrm{~cm}$ and $17 \mathrm{~cm}$ away from the center of the spectrometer in the measurement of the pulse readout mode 
and the current readout mode, respectively.

The events induced by neutrons and $\gamma$-rays are clearly distinguished from their pulse height distribution. The integrated charge of $\gamma$-rays is estimated to be less than $0.1 \%$ of that of neutrons owing to the high Q-value of the ${ }^{10} \mathrm{~B}(\mathrm{n}, \alpha){ }^{7} \mathrm{Li}$ reaction. Although the contamination of $\gamma$-rays to the neutron measurement in the current readout mode is small, the source were covered with a 2-mm-thick lead cap in the measurement of conversion factor to suppress the $\gamma$-ray contamination. The conversion factors which were obtained from ratio of count rates between pulse mode and current mode is 56.6 and 61.8 pulse/pC for the ${ }^{10} \mathrm{BF}_{3}$ counter and the ${ }^{\text {nat }} \mathrm{BF}_{3}$ counter, respectively.

\section{Experiment}

Figure 4 shows geometry of the pbar target station shielding structure. The station consists of anti-proton production target made of multiple layers of Inconel and copper disks and graphite dump with iron and concrete shielding. The simple structure and material of the station is suitable to provide benchmark data of neutron penetration. The anti-proton production target is irradiated with $120 \mathrm{GeV}$ proton beam provided from the Main Injector ring. The toroidal coil (TR105B) is placed just before the antiproton production target to monitor the beam intensity during this experiment. The typical time structure of the proton beam is $1.6 \mu$ s pulse width, with a cycle time of $3.0 \mathrm{~s}$. The power of the beam reached as high as $65 \mathrm{~kW}$ during our experiment. Downstream of the target, a graphite/aluminum dump is placed to absorb the remaining protons and secondary particles after the some extraction devices of produced anti-protons into the pbar extraction channel. These components are surrounded by $183-\mathrm{cm}$-thick iron and 122-cm-thick concrete shields. The area above these components is a restricted area during beam operation. The spectrometers of the ${ }^{10} \mathrm{~B}$ counter and the ${ }^{\text {nat }} \mathrm{B}$ counter were placed at two points that is $50 \mathrm{~cm}$ aside from the beam axis above the target shown in Fig.4. Activation samples of In, Al, $\mathrm{Cu}, \mathrm{Bi}$ were placed at the point on the beam axis above the target for the other measurement of neutron-induced activities after 10 days irradiation. ${ }^{11)}$

\section{Results}

Figure 5 shows a result of the MCS output of the ${ }^{10} \mathrm{BF}_{3}$ counter with the 110-mm-diameter moderator located above the target. The measured time profile of the current induced in the counter was clearly synthesized with each injection of pulsed beam obtained from TR105B. The mean current was obtained by integrating the pulse data which are subtracted with the background current which is $\sim 5 \mathrm{pA}$ due to detector leakage current. The neutron spectra were deduced using an unfolding code, SANDII ${ }^{17)}$, with the response function and an initial guess spectrum calculated by the MARS15 code. ${ }^{12)}$ The mean count rates of the ${ }^{10} \mathrm{~B}(\mathrm{n}, \alpha)^{7} \mathrm{Li}$ reaction in the counter were obtained by subtracting results of the ${ }^{\text {nat }} \mathrm{BF}_{3}$ counter from that of the ${ }^{10} \mathrm{BF}_{3}$ counter.

Figures 6 show the neutron energy spectra obtained together with the spectrum calculated by the MARS15 used

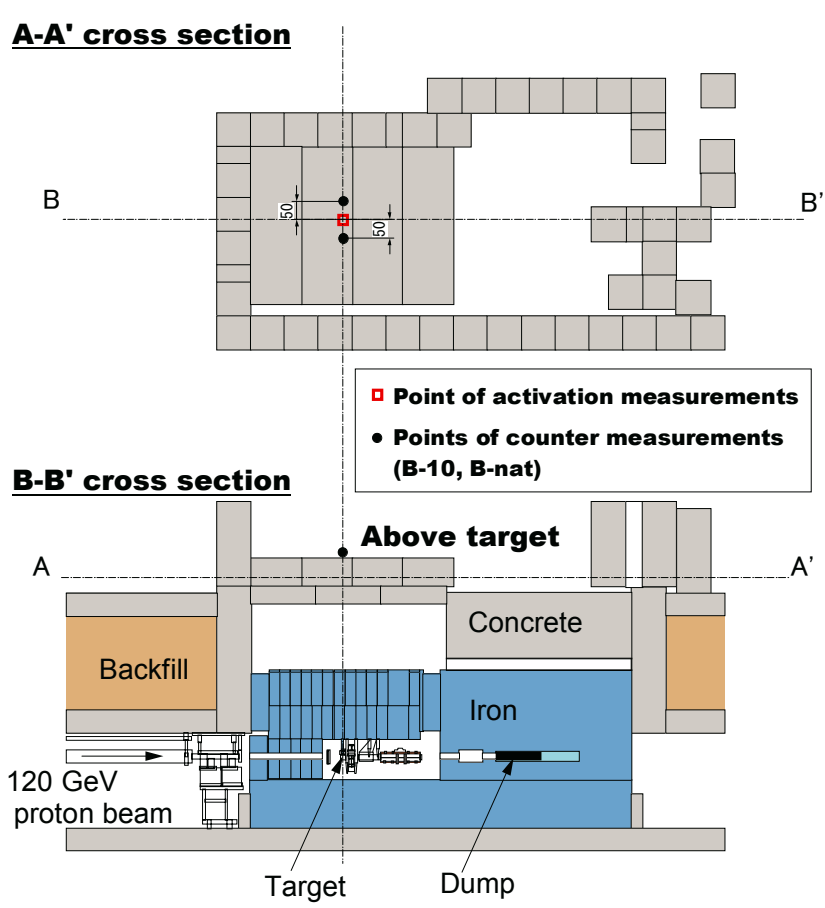

Fig. 4 Geometry of pbar target station

for the initial guess spectrum in the unfolding analysis. The

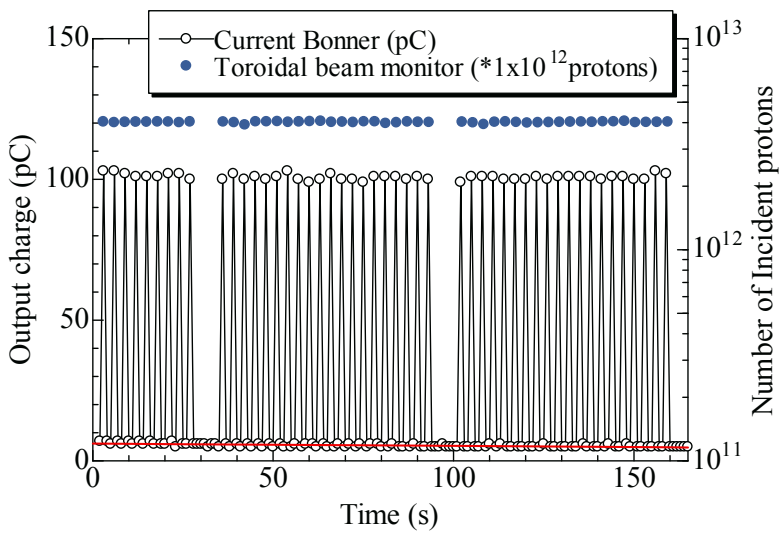

Fig. 5 MCS output obtained from ${ }^{10} \mathrm{BF}_{3}$ counter covered with 110-mm-thick moderator located above the target. The beam data of TR105B are also plotted.

measured spectrum has errors of the TR105R beam monitor $(10 \%)$ and the $\mathrm{I} / \mathrm{F}$ convertor $(10 \%)$, and the statistical errors $(9.3 \%)$. The total error of the neutron flux was estimated to be $17.1 \%$ without ambiguity of the unfolding method. The calculation generally agrees with the experimental results, but underestimates thermal neutron flux and slightly overestimates fast neutron flux above $1 \mathrm{MeV}$. The difference of thermal neutron flux which is drastically affected by surroundings and gaps in shielding would be due to ambiguities on the geometry description in the MARS calculation. The difference of fast neutron flux would be due to ambiguities of densities and compositions of materials described in the MARS calculation.

Figure 7 shows the comparison of reaction rates of nuclide between two experiments. Present results were deduced from the measured neutron spectra multiplying the corresponding 
activation cross-sections shown in ref.11. The reaction thresholds of ${ }^{115} \mathrm{In}\left(\mathrm{n}, \mathrm{n}^{\prime}\right),{ }^{27} \mathrm{Al}(\mathrm{n}, \alpha),{ }^{209} \mathrm{Bi}(\mathrm{n}, 4 \mathrm{n}),{ }^{209} \mathrm{Bi}(\mathrm{n}, 5 \mathrm{n})$, ${ }^{209} \mathrm{Bi}(\mathrm{n}, 6 \mathrm{n})$ are $0.6,3.3,22.6,29.6,38,45.3 \mathrm{MeV}$, respectively. Preset results show in good agreement with the activation results within $30 \%$ in the energy range.

\section{Conclusion}

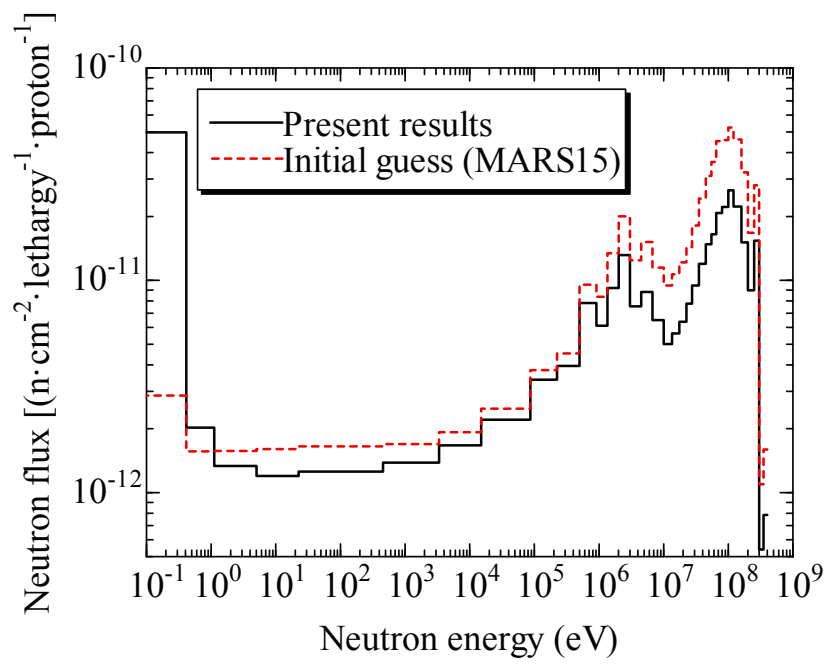

Fig. 6 Neutron energy spectra above the target unfolded with the initial spectrum calculated by the MARS code

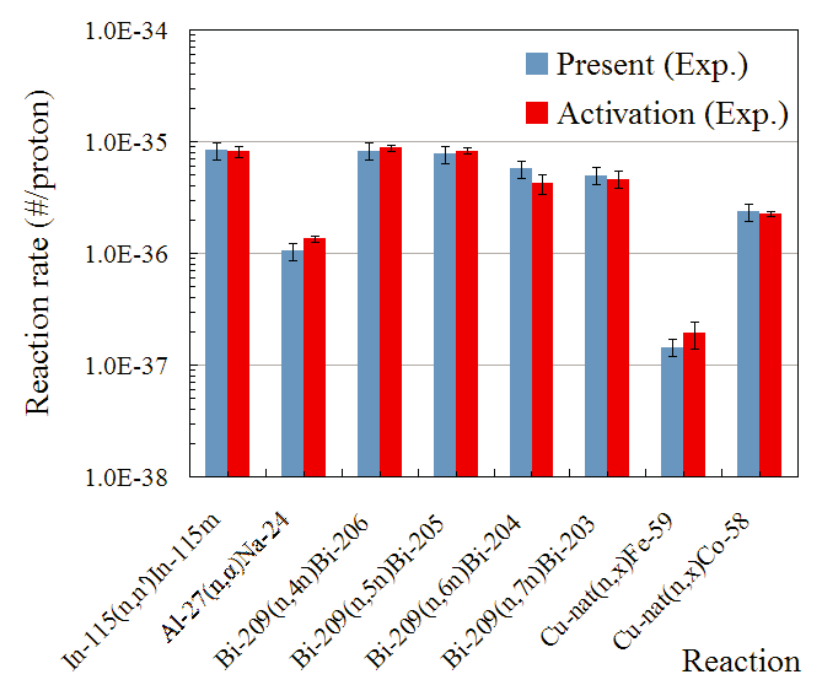

Fig. 7 Comparison of reaction rates of nuclides between present results and activation results

We have developed a current readout Bonner sphere technique to measure neutron spectra in radiation bursts at the pbar target station by a multi-moderator spectrometer (Bonner spheres) using a pair of $\mathrm{BF}_{3}$ proportional counters filled with different ${ }^{10} \mathrm{~B}$ isotope enrichment gases, ${ }^{\text {nat }} \mathrm{BF}_{3}$ (18\% enrichment) and ${ }^{10} \mathrm{BF}_{3} \quad(96 \%$ enrichment $)$ and measured the neutron energy spectra behind $183-\mathrm{cm}$-thick iron and 122-cm-thick concrete shields from $120 \mathrm{GeV}$ proton induced reactions. Present result was consistent with the other experimental results by the activation method which shows reliability of both data. The initial guess spectrum calculated by MARS 15 show slightly overestimation in $\mathrm{MeV}$ region and underestimation in the thermal energy region, which would be due to ambiguities on the geometry description in the MARS calculation should be investigated further.

The current readout Bonner sphere technique would be helpful to measure shielding benchmark data in high energy proton accelerators under the intense burst field that is impossible to use active counter in pulse mode. Although the lack of sensitivity for over $10 \mathrm{MeV}$ neutrons should be improved in further experiment, the spectrum results would be helpful to investigate particle interaction and transport codes for high energy region.

\section{Acknowledgment}

This work is supported by grand-aid of ministry of education (KAKENHI 19360432) in Japan. Fermilab is a U.S. Department of Energy Laboratory operated under Contract DE-AC02-07CH11359 by the Fermi Research Alliance, LLC. This work was partially performed under the Common-Use Facility Program of JAEA.

\section{References}

1) J. S. Hendricks, et al., "MCNPX 2.6.0 Extensions," LA-UR-08-2216, Los Alamos National Laboratory (LANL), (2008).

2) A. Ferrari, P. R. Sala, "Intermediate and high energy models in FLUKA: improvements, benchmarks and applications,' Proc. Int. Conf. on Nucl. Data for Science and Technology, Ed. G. Reffo, A. Ventura and C. Grandi (Bologna: Italian Phys. Soc.), NDST-97, 59[I], 247 (1997).

3) N. V. Mokhov, "The Mars Code System User's Guide," Fermilab-FN-628, Fermi National Accelerator laboratory (FNAL), (1995), N. V. Mokhov, S. I. Striganov, "MARS15 Overview," Fermilab-Conf-07-008-AD, Fermi National Accelerator laboratory (FNAL), (2007).

4) H. Iwase, K. Niita and T. Nakamura, "Development of general-purpose particle and heavy ion transport Monte Carlo code," J. Nucl. Sci. Technol., 39[11], 1142 (2002).

5) N.Nakao et al., Nucl. Instrm. Meth. B 266, 93 (2008).

6) G. R. Stevenson et al., Health Phys., 43, 13 (1982).

7) S. Ban et al., Nucl. Instum. Methods, 174, 271 (1980).

8) M.Awschalom, et al., Nucl.Instrum.Meth. 136, 521 (1976).

9) H.Nakashima, et al., Proc. of the Shielding Aspects on Accelerator, Target and Irradiation Facilities -SATIF6, SLAC, Apr. 10-12, 2002, p. 27 (2002).

10) H. Nakashima, et.al, Nucl. Technol., 168, 482(2009)

11) H. Yashima et al., Proc. of fifth International Symposium on Radiation Safety and Detection Technology (ISORD-5), Kitakyushu, Japan, June. 15-17, 2009 to be published.

12) T. Sanami et al,"Applicability of a Bonner sphere technique for neutron spectrum measurements in a pulsed neutron field of the $120-\mathrm{GeV}$ proton facility", Proc. of $22 \mathrm{nd}$ workshop on Radiation Detectors and Their Uses, Tsukuba, Japan, Feb. 5-7, 2008, p.148(2008).

13) Y.Uwamino et al., Nucl. Instrm. Meth. A 239, 299 (1985).

14) M. B. Chadwick et al., "Cross-section evaluations to $150 \mathrm{MeV}$ for accelerator-driven systems and implementation in MCNPX,'” Nucl. Sci. Eng., 131, 293 (1999).

15) Y. Tanimura et al., "Construction of Monoenergetic Neutron Calibration Fields Using ${ }^{45} \mathrm{Sc}(\mathrm{p}, \mathrm{n}){ }^{45} \mathrm{Ti}$ Reaction at JAEA", 
Radiat. Prot. Dosim., 126, 8 (2007).

16) K. Iijima et al, Proc. of fifth International Symposium on Radiation Safety and Detection Technology (ISORD-5), Kitakyushu, Japan, June. 15-17, 2009 to be published.
17) W. McElroy, S. Berg, T. Crocket, G. Hawkins, "A computer automated iterative method for neutron flux spectra determination for foil activation," AFWL-TR-67-41 (1967). 